\title{
A Review: Cloud Computing using Various Task Scheduling Algorithms
}

\author{
Pirtpal Singh \\ M.Tech Scholar \\ Sri Guru Granth Shaib World University \\ Fatehgarh Sahib
}

\author{
Navpreet Kaur Walia \\ Assistant Professor \\ Sri Guru Granth Shaib World University \\ Fatehgarh Sahib
}

\begin{abstract}
Cloud Computing discusses to the use of computing, infrastructure, software, as a service. It's a procedure of utility computing where the consumer need not own the essential infrastructure and pay for only what they use. Computing resources are transported as virtual machines. Proficient task scheduling apparatus can meet users' necessities, and advance the resource utilization, thereby improving the overall performance of the cloud computing environment. The role of task level scheduling in power organisation on multicore multiple voltage embedded systems. Task scheduling algorithms play an imperative role where the aim is to schedule the tasks efficiently so as to condense the improvement time and advance resource utilization. This paper represents two scheduling algorithms for scheduling tasks taking into attention their computational complexity and computing capacity of processing basics. Prior's comparison studies have recurrently operated with shortening assumptions, such as independent tasks, affectedly generated difficulties or the assumption of zero communication delay.
\end{abstract}

\section{Keywords}

Cloud Computing, Task Scheduling, Infra-Structure, Software as-a- service and Scheduling Algorithms.

\section{INTRODUCTION}

Cloud computing is the latest exhortation in the IT industry. It is an emerging computing prototype with fundamentals of grid computing, utility calculating, service concerned with architecture, virtualization and web 2.0[1]. The user can access all essential hardware, platform, software, applications, infrastructure and storage with the possession of just an internet construction. "A Cloud is a type of comparable and dispersed system consisting of a collection of inter-related and virtualized computers that are enthusiastically provisioned and presented as one or more shared computing resources based on service level provisions established through co-operation between the service provider and consumers". Several of the presentations of cloud computing are community networking, on-line gaming, and scientific applications. One of the key problems in public clouds is that of security and confidentiality. In public clouds data centres hold end-users data which then would have been stored on their own computers. Hence there is an increasing demand for private clouds. A private cloud is one which is possessed and operated within the firewalls of an organization [2].

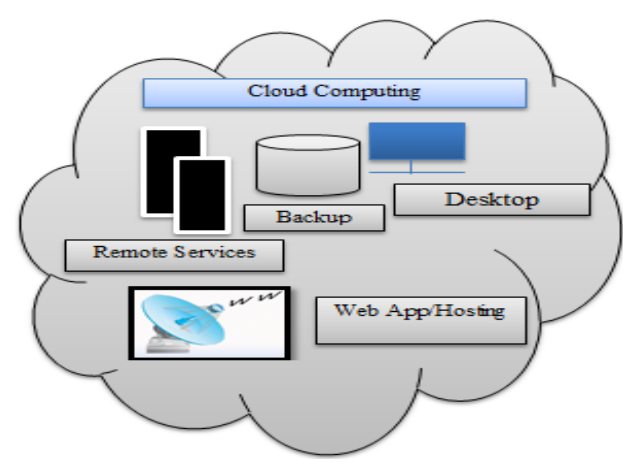

Fig no: 1 Cloud computing

\section{A. Scheduling}

Scheduling discusses to the set of rules to control the order of work to be achieved by a computer system. There have been several types of scheduling algorithm existing in disseminated computing system, and job scheduling is one of them. The main benefit of task scheduling algorithm is to attain a high performance calculating and the best system throughput. Scheduling achieves availability of CPU memory and good arrangement policy [3] gives maximum utilization of resource. We associated three algorithms Time Shared, Space shred and generalizes priority algorithm. Scheduling a set of reliant on or independent tasks for similar implementation on a set of mainframes is an imperative and computationally complex difficult.

\section{B. $\quad$ Task Scheduling}

Task scheduling has been about for many years and provides essential provision for both grid computing and cloud computing to growth the system performance and competence [4]. In cloud computing and grid computing environment, the computing possessions can be varied in many aspects which provide suitable conditions to implement applications with multiple inherent parallelism. The aim of task scheduling is to assign tasks to available processors such that preference necessities between tasks are satisfied and the overall time compulsory to execute all tasks, the make span, is minimized.

\section{VARIOUS TECHNIQUES OF TASK SCHEDULING}

Tasks are submitted to a server through a client terminal which is also in charge of initializing the [8] tasks and generating task evidence table including task number, storing space required, task type, receive time, etc. Along with the tasks, task information table will be delivered to the scheduling server. 


\subsection{Efficient Task Scheduling Algorithm [5]}

In this paper describes the efficient algorithms like Static scheduling algorithms reflect that all tasks arrive at the same immediate of time and they are autonomous of the system supply's states and their accessibility. The static heuristics include the basic simple scheduling approaches like First Come First Serve and Round Robin methods. FCFS methods assembles the tasks and queues them until resources are presented and once they become available the tasks are allocated to them based on their arrival time. It is less complex in nature but does not consider any other criteria for scheduling the tasks to machines.

\subsection{RAS: Scheduling Algorithm Based on \\ Resource Attribute Selection in a Task \\ Scheduling Framework [5]:}

These are considered the FCFS for parallel processing and are aiming at the reserve with the smallest waiting queue time and are selected for the incoming task. The disadvantages of FCFS are that it is non-pre-emptive. The shortest tasks which are at the back of the queue have to delay for the long task at the front to finish .Its improvement and response is quite low.

\begin{tabular}{|c|c|c|c|}
\hline Process & Arrival Time & Execute Time & Service Time \\
\hline P0 & 0 & 5 & 0 \\
P1 & 1 & 3 & 5 \\
P2 & 2 & 8 & 8 \\
P3 & 3 & 6 & 16 \\
\hline
\end{tabular}

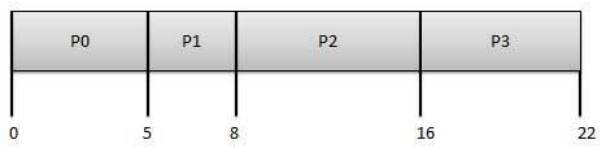

Fig no: 2 FCFS Algorithm

\subsection{Performance study Algorithm [5]:}

Mentioned in Round Robin algorithm focuses on the fairness. $\mathrm{RR}$ uses the ring as its queue to store jobs. Each job in a queue has the same performance time and it will be executed in turn. If a job can't be completed during its turn, it will be deposited back to the queue waiting for the next turn. The advantage of $\mathrm{RR}$ algorithm is that each job will be executed in chance and they don't have to be waited for the previous one to get finished. But if the load is found to be weighty, RR will take a long time to complete all the jobs.

Quantum $=3$

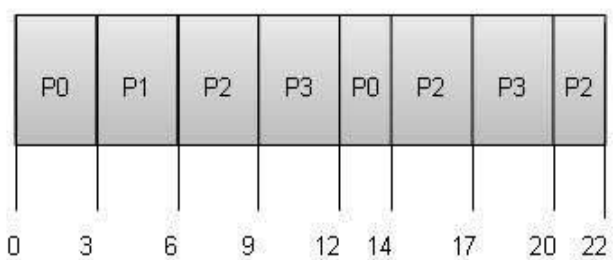

\subsection{Cloud Task Scheduling based techniques [8]}

ACO algorithm is an accidental exploration algorithm, like other evolutionary algorithms. It replacements the performance of real ant colonies in nature to search for food and to attach to each other by pheromone laid on paths covered. Many investigators used ACO to solve NP-hard difficulties such as traveling salesman problematic, graph colouring problem, vehicle routing problem, and so on. Load Balancing Ant Colony Optimization algorithm to find the optimum standby allocation for each task in the dynamic cloud system. Not only does it minimize the kinds pan of a given tasks set but it also adapts to the active cloud computing system and balance the complete system load.

\subsection{Agent Based Task Scheduling [9]}

Derived Min-Min and Max-Min Algorithms are two other heuristic methods used for task scheduling. Min-min heuristic selects the lowest task first from all the available tasks and gives it to a machine which gives the minimum completion time for that task. It growths the total completion time of all the tasks and hence increases the make span. But it does not consider load of the machines before scheduling as simply conveying smaller tasks on faster machines. Here the predictable completion time and completing time for a task are considered to be almost same values or close values. The long tasks have to wait for finalizing the execution of smaller ones. But the method improves the system's overall quantity.

\subsection{Analysis Methods [10]}

Described as Genetic Algorithm and Imitation Forging are two other general methods in heuristic approach which is used to complete near optimal scheduling. In Genetic Algorithm approach we complete four changed operations, evaluation, selection, cross over and mutation. The initial populace represents the possible mappings of the given task list on the presented machines. Each job is characterized as a vector in which each position of that vector characterises a task in the task list. The value in each position characterizes the machine to which the task is planned. Each job represents a chromosome. Every chromosome has a fitness value indicating the overall execution time of all the tasks which is formed from the mapping of tasks to resources constituting that gene and it is selected such that it reduces make span. This method uses past results with present results to get better possible mappings and survival of the fittest takes place.

Fig no: 3 Round Robin Algorithm

Table no: 1 Comparison of Existing Algorithms

\begin{tabular}{|l|l|l|l|}
\hline Scheduling Techniques & Parameters Considered & Advantages & Disadvantages \\
\hline $\begin{array}{l}\text { First Come First Serve } \\
\text { (First In First Out } \\
\text { Technique) }\end{array}$ & Arrival Time & Easy to implement & $\begin{array}{l}\text { Doesn't consider any other } \\
\text { criteria for scheduling }\end{array}$ \\
\hline $\begin{array}{l}\text { Round Robin } \\
\text { (Time Slot Based Multi- } \\
\text { Threading Technique) }\end{array}$ & Arrival time, Time quantum & $\begin{array}{l}\text { Less complexity and load is } \\
\text { balanced more fairly }\end{array}$ & Pre-emption is required \\
\hline
\end{tabular}




\begin{tabular}{|l|l|l|l|}
\hline $\begin{array}{l}\text { Min-Min, Max Min } \\
\text { (Task Queue Prioritization } \\
\text { Technique) }\end{array}$ & $\begin{array}{l}\text { Make span, Expected } \\
\text { completion time }\end{array}$ & $\begin{array}{l}\text { Better make span compared } \\
\text { to other algorithms }\end{array}$ & $\begin{array}{l}\text { Poor load balancing and } \\
\text { QoS factors are not } \\
\text { considered }\end{array}$ \\
\hline $\begin{array}{l}\text { Genetic Algorithm } \\
\text { (Fitness Solution Based } \\
\text { Technique) }\end{array}$ & $\begin{array}{l}\text { Make span, Efficiency, } \\
\text { Performance, Optimization }\end{array}$ & $\begin{array}{l}\text { Better performance and } \\
\text { efficiency in terms of make } \\
\text { span }\end{array}$ & $\begin{array}{l}\text { Complexity and long-time } \\
\text { consumption }\end{array}$ \\
\hline
\end{tabular}

\section{CONCLUSION}

Efficient scheduling algorithms always play a significant role in the performance provided by a cloud computing system. A study of existing task scheduling algorithms is done in this paper. It considers some heuristic, energy efficient and hybrid methods for study. A brief analysis of each method is done and most algorithms perform scheduling based on one or two parameters. A better scheduling algorithm can be developed from the existing methods by adding more number of metrics which can result in good performance and outputs that can be deployed in a cloud environment in future. The table created, consolidates all the different scheduling parameters used in the existing scheduling algorithms. A good scheduling algorithm must consider the requirements of users satisfying their needs provided in SLA and at the same time beneficial to the cloud providers. Combining different parameters such that to obtain an efficient scheduling algorithm and improve the overall performance of the cloud services can be done as an enhancement. An Advantage of task scheduling is Effective use of all AIS resources; User Deadline met and better use of multiprogramming capabilities. Traditional approaches of scheduling lead to high response time and low throughput.

\section{REFERENCES}

[1] Raja Manish Singh, Sanchita Paul, and Abhishek Kumar. "Task Scheduling in Cloud Computing: Review." International Journal of Computer Science and Information Technologies 5.6 (2014): 7940-7944.

[2] Er Shimpy, and Mr Jagandeep Sidhu. "Different Scheduling Algorithms In Different Cloud Environment." algorithms 3.9 (2014).

[3] Mekkittikul, Adisak, and Nick McKeown. "A practical scheduling algorithm to achieve 100\% throughput in input-queued switches." INFOCOM'98. Seventeenth Annual Joint Conference of the IEEE Computer and Communications Societies. Proceedings. IEEE. Vol. 2. IEEE, 1998.
[4] Shital Patil, et al. "Performance improvement in cloud computing through dynamic task scheduling algorithm." Next Generation Computing Technologies (NGCT), 2015 1st International Conference on. IEEE, 2015.

[5] S.Sindhu, and Saswati Mukherjee. "Efficient task scheduling algorithms for cloud computing environment." High Performance Architecture and Grid Computing. Springer Berlin Heidelberg, 2011. 79-83.

[6] Zhao, Yong, et al. "RAS: A Task Scheduling Algorithm Based on Resource Attribute Selection in a Task Scheduling Framework." Internet and Distributed Computing Systems. Springer Berlin Heidelberg, 2013. 106-119.

[7] Jin, Shiyuan, Guy Schiavone, and Damla Turgut. "A performance study of multiprocessor task scheduling algorithms." The Journal of Supercomputing43.1 (2008): 77-97.

[8] Li, Kun, et al. "Cloud task scheduling based on load balancing ant colony optimization." Chinagrid Conference (ChinaGrid), 2011 Sixth Annual. IEEE, 2011.

[9] Ding, Shun-Li, Jing-Bo Yuan, and Jiu-Bin Ju. "An algorithm for agent-based task scheduling in grid environments." Machine Learning and Cybernetics, 2004. Proceedings of 2004 International Conference on. Vol. 5. IEEE, 2004.

[10] Mathew, Tojo, K. Chandra Sekaran, and Jithin Jose. "Study and analysis of various task scheduling algorithms in the cloud computing environment."Advances in Computing, Communications and Informatics (ICACCI, 2014 International Conference on. IEEE, 2014. 